\title{
The Regimes of Translational and Transtextual Representation
}

English version

David Palumbo-Liu

\section{(2) OpenEdition Journals}

Electronic version

URL: http://journals.openedition.org/transtexts/170

DOI: $10.4000 /$ transtexts. 170

ISSN: 2105-2549

Publisher

Gregory B. Lee

Printed version

Date of publication: 1 May 2006

Number of pages: 37-39

ISSN: 1771-2084

Electronic reference

David Palumbo-Liu, "The Regimes of Translational and Transtextual Representation », Transtext(e)s Transcultures 跨文本跨文化 [Online], 1 | 2006, Online since 13 September 2009, connection on 24 April 2019. URL : http://journals.openedition.org/transtexts/170 ; DOI : 10.4000/transtexts. 170 


\section{The Regimes of Translational and Transtextual Representation BY David Palumbo-Liu}

It was probably during the Romantic era that a particularly modern sense of translation appeared. Typified by Charles Baudelaire's famous address at the start of Les Fleurs du Mal, "Hypocrite lecteur, mon sembable, mon frère," we find embedded a notion that both reading and writing are haunted by a moral, and immoral, doubleness. Delicious and irresponsible at once, the transcriptions of the imagination transgressed at once both the notion that only God would be the great creator and point of both reverence and reference, and that there was a sole truth to be pointed to or expressed. Either in writers' taking on for themselves the graphic signature of creation or in a reader's belated and yet original reading of that expression and its transcoding into another subject's repertoire of representation, the arena of writing and reading became newly complex and dialogic. Hence the notion of translation became at least loosely unanchored from both its linguistic, epistemological and ethical obligation to be "true to the original," since the idea of art and truth had become radically rescripted and the concept of originality with it.

It may not be an accident that these aesthetic revolutions took place with the flourishing of the bourgeoisie and a sense of individual's entitlement. And yet, returning to Baudelaire, there is the well-known tension between the aspirations of the individual toward absolute freedom and the registering of the persistent claims of the social and historical. That tension is well expressed in his seminal essay on the modern, "The Painter of Modern Life," in which the modern is constantly under erasure by the flow of time and yet reconsolidated within something called the "classic." Let us imagine then that this is not only an aesthetic division between creativity and tradition, or a temporal one between the present and the past, but also a social one between the individual and the collective. I want to consider this inaugural issue of Transtext(e)s Transcultures as engaging, in a strong way, this last element, and I add here the issue of power.

Each of the essays I have perused in some way or another addresses the question of power in transtextual and translational activity. The power of the second text (and I use this designation simply because it seems the most neutral--I want to insist that this is a cardinal, not ordinal designation, without any sense of belatedness or inferiority) to stand in for the other text as its representative, even shrouded as it is in debt. This power is not a given, nor is it won easily. And it is not permanent and irrevocable. It is rather contingent upon precisely its sociopolitical and historical context. Getting back, yet again, to Baudelaire, the question is how the individual instance is articulated in social space. For instanceRatheesh Radhakrishnan's examination of cinematic "translation" in Indian films points out how the Malayalam adaptation of Aithe recontextualizes and reinvents the film in a radically 
different class context; the essay discloses the ways the narrative of the former film had to be adapted to an entirely different social and political world. And yet what is most fascinating in this discussion is not the recontextualization nor the rescripting alone, but the ways that the narrative and cinematic logic of the former film and that of the adaptation show, in their disjunctions, not only the movement of history but also its power in shaping the ethical, aesthetic and sentimental repertoires of viewing publics. If a strong, material sense of history is foregrounded in Radhakrishnan's essay, then the abstraction of space and time, and their translation into the logos of geographic and ethnographic discourse is emphasized in Pelletier's essay on Elisée Reclus. This essay finds the workings of discursive power at the most general level - the segmenting of East and West, facilitated by the work of a scholar whose expertise in "The Orient" was not lessened by the fact that he had actually never visited it. The epistemological and the empirical thus stand in an uneasy tension, and that tension is resolved only through the workings of desire and power- the predispositions of the West to "see" the Orient in a certain fashion, and the accretive power of discourse networks to produce a specific reality effect.

Florent Villard's essay similarly focuses on the disjunctures of real space and time, in a fascinating account of the contemporary production of "China"-at once rendered into the past, and yet in which the past is transformed into the most "realistic" frame for the present. Simply regarding this as the commodification of the nation only goes so far in explaining the specific forces of erasure and reconstitution, as Villard demonstrates. It raises the question again of audience, and the assumptions that feed into the production of "China"- how does the state imagine the non-Chinese world to (want to) see it? What dimensions, shapes, details, of the past seem to blend into that perceived object of desire, and what does this tell us not only about the Chinese production of the past/present nation, but also about the ways in which the West has, explicitly or implicitly, instantiated its tastes, desires, appetites?

Representation is again at the heart of Marie-Eve Blanc's comparative study of the representations of Vietnamese immigrants in both the French and Canadian press. Her study shows how the difference in representation (as, respectively, the positive image of multicultural migration and the negative image of criminality and contamination) depends on the larger social and cultural contexts of immigration. The essay explains how the historical context behind these different representations situates Asia and the foreign within different nation-state practices and politics.

Stéphanie Tsai's study of the reception of André Malraux in Taiwan gives us an excellent example of how the same figure can be very differently received outside their home country. Most strikingly, Tsai shows how different predispositions influence the assessment of whether or not Malraux's notion of a global community could be grasped fully. Are his terms translatable into Chinese ? Is there a huge conceptual gulf between the languages and cultures? Or was this an historical failure, rather than a linguistic one? Finally, Corrado Neri's study similarly addresses the idea of influence, noting the affinities between the cinematic project of Tsai Ming-liang and that of François Truffaut. Neri argues that the intertextual elements in Taiwanese cinematographer's films, conspicuously referencing Truffaut, take on a spiritual resonance as well, as both film-makers are obsessed with the idea of freedom. The distance between the French New Wave and contemporary Taiwanese film-making is thus shortened dramatically. The author does a fine job of using this affinity to reveal the special qualities of Tsai's films, in contradistinction to the manners in which other film-makers such 
as Hou Hsiao-hsien and Yang Dechang have employed allusions to European and American films. It would be fascinating to press further the ways in which the different historical contexts in which Truffaut and Tsai work inflect the notion of "freedom" quite differently.

In the case of the precise location of this publication, in the interstices of Asia and Europe and radiating out and inwards, the "modern" context has not dissipated, but it has been problematized. While the issues of power raised at the start of this short contribution have remained, the essays contained in this issue, and hopefully others to come, have demonstrated that the increased flows of migration, the fissures in local and regional identities brought about by increased globalization, the varied receptions of the "foreign" into the "domestic," have produced much more complex configurations of power-the transportability, the transcodability of texts is dependent now upon often contradictory aims and interests. 1 If I were to make a modest suggestion in this regard, it would be to see how the regimes of translational and transtextual representation are imbricated in a dialectical tension between the residual claims of the autonomy of art and the sociopolitical and historical contingencies that may block, facilitate, force, divert, or erase the "trans"-position of texts. It would then be not a sense of moral indignation or aesthetic propriety (call it "taste") that would drive us to track the transmutation of texts into other texts, but rather it would be both political and historical sensitivity and commitment that would engage our critical attention.

\title{
Les régimes \\ de la représentation transnationale et transtextuelle
}

\begin{abstract}
C'est probablement pendant la période romantique qu'apparut une idée spécifiquement moderne de la traduction. Comme l'illustre la célèbre apostrophe de Charles Baudelaire au commencement des Fleurs du mal, « - Hypocrite lecteur, - mon semblable, - mon frère ! ", ''idée que lire et écrire sont hantés par un doublé moral et immoral est bien ancrée. Délicieuses et irresponsables, les transcriptions de l'imagination transgressaient à la fois la notion que seul Dieu était le grand créateur et le point de référence et de révérence, et celle qu'il n'existait qu'une seule vérité à indiquer et à exprimer. Ainsi l'arène de l'écriture et de la lecture se complexifia et devint plus dialogique, que ce soit lorsqu'un écrivain s'appropria la signature graphique de la création ou qu'un lecteur apporta sa lecture tardive, mais toutefois originale, de cette expression et la transcoda dans le répertoire de représentation d'un autre sujet. Et dès lors la notion de traduction se vit quelque peu déliée de son obligation linguistique, épistémologique et éthique de " coller à l'orignal ", puisque l'idée de l'art et de la vérité avait été totalement réécrite et avec elle, le concept d'originalité.
\end{abstract}

1 For my early meditation on these issues, see "Unhabituated Habituses" in David Palumbo-Liu \& Hans Ulrich Gumbrecht (eds.), Streams of Cultural Capital, Stanford, CA : Stanford University Press, 1997. 
Ce n'est pas par hasard que ces révolutions esthétiques coïncidèrent à l'avènement de la bourgeoisie et d'un sens du droit de l'individu. Et pourtant, si l'on en revient à Baudelaire, on trouvera la tension familière entre la soif de liberté absolue de l'individu et sa reconnaissance des contraintes sociales et historiques permanentes. Cette tension est bien décrite dans son essai déterminant sur le moderne intitulé « Le peintre de la vie moderne », dans lequel le moderne est effacé en permanence par le passage du temps pour être reconsolidé à l'intérieur de ce qu'on appelle le «classique ». Imaginons un instant qu'il ne s'agisse pas là uniquement d'une division esthétique entre créativité et tradition, ou temporelle entre le présent et le passé, mais aussi d'une division entre l'individu et le collectif. Je voudrais voir dans ce premier numéro de Transtext(e)s Transcultures une négociation de ce dernier élément, auquel j'ajouterais ici la question du pouvoir.

En effet, chacun des essais que l'on m'a transmis traite d'une manière ou d'une autre de la question du pouvoir dans les activités transtextuelles et de traduction. Le pouvoir du second texte (et j'utilise ici ce terme uniquement parce qu'il me semble être le plus neutre - je tiens à souligner qu'il s'agit d'un terme cardinal et non pas ordinal, sans aucune connotation de retard ou d'infériorité) à servir de représentant à l'autre texte, même s'il lui est redevable. Ce pouvoir n'est pas une évidence et ne s'obtient pas facilement. De plus, il n'est ni permanent ni irrévocable. II dépend précisément de son contexte historique et sociopolitique. Pour en revenir une fois encore à Baudelaire, le problème est de savoir comment le cas individuel s'articule dans l'espace social. Par exemple, l'examen de la «traduction » cinématique dans les films indiens par Ratheesh Radhakrishnan démontre comment l'adaptation de Aithe par Malayalam replace et réinvente le film dans un contexte de classe radicalement différent; l'essai dévoile comment le narratif du premier film a dû être adapté à un monde social et politique complètement différent. Pourtant, ce qui fascine dans ce débat ce n'est pas seulement la recontextualisation ni la réécriture, mais la manière dont la logique narrative et cinématique du premier film et celle de l'adaptation montrent, dans leur disjonctions, non seulement le mouvement de l'histoire mais aussi son pouvoir dans la formation des répertoires éthique, esthétique et sentimental du public. Si l'on met en avant un sens matériel très fort de I'histoire dans l'essai de Radhakrishnan, celui de Philippe Pelletier sur Elisée Reclus privilégie l'abstraction de l'espace et du temps, ainsi que leur traduction dans le logos du discours géographique et ethnographique. Cet essai démontre que les rouages du pouvoir discursif au niveau le plus général - à savoir la segmentation Est-Ouest - peuvent s'aider de l'œuvre d'un savant dont l'expertise dans le domaine de «l'Orient » n'est en rien réduite par le fait qu'il n'y soit jamais allé. L'épistémologique et l'empirique s'affrontent donc dans une difficile tension qui ne peut être résolue qu'au moyen des rouages du désir et du pouvoir - la prédisposition de l'Occident à « voir » l'Orient d'une certaine façon, et le pouvoir accru des réseaux de discours à produire un effet de réalité spécifique.

De même, l'essai de Florent Villard s'intéresse aux disjonctions du temps et de l'espace réel dans une description fascinante de la production contemporaine de la «Chine»immédiatement transposée dans le passé, mais le passé lui-même étant transformé en cadre « réaliste » du présent. Villard démontre que n'y voir qu'une commodification de la nation n'explique que partiellement les forces d'effacement et de reconstitution. Cela pose de nouveau la question du public, et des présuppositions qui s'introduisent dans la production de «la Chine». Quelles dimensions, quelles formes, quels détails du passé se mêlent-ils dans cet objet perçu du désir, et qu'est-ce que cela nous dit non seulement de la production 
chinoise de la nation passée/présente, mais aussi des façons dont l'Occident a, explicitement ou non, représenté ses goûts, ses désirs et ses appétits?

La représentation est encore une fois au centre de l'étude comparative menée par MarieEve Blanc sur les représentations des immigrants vietnamiens dans la presse canadienne et française. Ses recherches démontrent que la différence de représentation (image positive de la migration multiculturelle ou image négative de la criminalité et de la contamination) dépend des contextes sociaux et culturels de l'immigration. Cet essai explique comment le contexte historique qui sous-tend ces différentes représentations situe l'Asie et l'étranger à l'intérieur de différentes pratiques et politiques d'état-nation.

L'étude de Stéphanie Tsai sur la réception d'André Malraux à Taiwan démontre parfaitement comment le même personnage peut être reçu de manière totalement différente en dehors de son pays. Ce qui est peut-être le plus frappant c'est que Tsai démontre comment les différentes prédispositions ont influencé l'opinion que la notion de Malraux d'une communauté globale puisse être comprise ou non. Les termes qu'il emploie peuvent-ils être traduits en chinois ? Existe-t-il un océan conceptuel entre les langues et les cultures ? Ou s'agissait-il d'un échec historique plutôt que linguistique ? Enfin, l'étude de Neri aborde de même l'idée d'influence, en soulignant les affinités entre le projet cinématique de Tsai Ming-liang et celui de François Truffaut. Neri affirme que les éléments intertextuels des films du cinématographe taïwanais, faisant manifestement référence à Truffaut, ont de plus un écho spirituel, puisque les deux cinéastes sont obsédés par l'idée de la liberté. La distance séparant la Nouvelle Vague française du cinéma taiwanais contemporain en est d'autant plus raccourcie. L'auteur emploie judicieusement cette affinité pour démontrer les qualités spéciales des films de Tsai, contrairement à la manière dont d'autres cinéastes tels Hou Hsiao-hsien et Yang Dechang ont utilisé des allusions à des films européens et américains. II serait fascinant de rechercher plus avant comment les différents contextes culturels dans lesquels travaillent Truffaut et Tsai ont décliné différemment la notion de liberté.

En ce qui concerne la situation précise de cette publication, dans les interstices de l'Asie et de l'Europe et rayonnant vers l'extérieur mais aussi vers l'intérieur, le contexte «moderne » ne s'est pas dissipé, mais problématisé. Alors que demeurent les questions de pouvoir évoquées au début de ce bref article, les essais contenus dans ce numéro ont apporté la preuve que l'augmentation des flux migratoires, les fissures provoquées par la mondialisation dans les identités régionales et locales, les réceptions variées de " l'étranger " dans le "domestique", ont produit des configurations bien plus complexes du pouvoir - la transportabilité, la transcodabilité des textes dépend à présent souvent de finalités et d'intérêts contradictoires. ${ }^{2}$ Ma modeste suggestion à ce sujet, serait de considérer comment les régimes de représentation transtextuelle et de traduction s'imbriquent dans une tension dialectique entre les exigences résiduelles de l'autonomie de l'art et les contingences sociopolitiques et historiques qui pourraient bloquer, faciliter, détourner, ou encore effacer la "trans "-position des textes. Ce ne serait pas alors un sens d'indignation morale ou de bienséances esthétiques (le " goût ») qui nous pousserait à suivre la transmutation de textes en d'autres textes, mais plutôt une sensibilité à la fois historique et politique et un engagement qui mobiliserait notre attention critique.

2 En ce qui concerne mes premières méditations à ce sujet, voir "Unhabituated Habituses" in David Palumbo-Liu \& Hans Ulrich Gumbrecht (eds.), Streams of Cultural Capital, Stanford, CA : Stanford University Press, 1997. 


\section{翻译与跨 \\ 文本表征的机制}

大概是在浪漫主义运动时期, 出现了翻译在现代意义上的独特含义。以波德莱 尔 (Baudelaire) 在《恶之花》卷首的名言为代表, “伪善的读者, 我的同伴, 我的兄弟”，我们发现其中内含着一种的观念，即无论是阅读还是写作都会被 一种道德和非道德所困扰, 具有双重性。既美妙有趣而又不值得信赖, 想象的 转写同时违背了这样的观念, 只有上帝才可能是伟大的创造者, 是令人崇敬与 参照的顶点, 只存在唯一一种可以指向或表达的真理。或者在一位作家为其自 身对创造性的类似图画的签名的接受中，或者在一名读者迟后而具独创性的对 那种表达及其成为另一个主体有关表征的全部技能的转码的阅读中，写作与阅 读的空间新近变得复杂而又可以对话了。因此, 翻译的观念, 从其语言、认识 论和伦理道德方面的义务到 “忠实于原创” 方面, 至少已经变得松散而不稳定 了, 因为艺术与真实的思想已经基本变成为复写和与之相随的独创性的观念 了。

这些审美革命与资产阶级和一种个体权利意识的繁荣同时出现, 或许并非意 外。可是回到波德莱尔来说, 在向往绝对自由的个体渴望和对社会与历史持久 稳固的主张之流露表达间有一种为人熟知的张力。那种张力在其有关现代人的 开创性的文章《现代生活的画家》中得到了很好的表达, 在此文中, 现代人 一直处于被时间流所擦除的状态, 而又在所谓的 “经典作品” 中得以重新巩固 与强化。那么我们设想一下，这不仅仅是创造性与传统之间的一种审美分化， 或在当前与过去之间的一种时间分化，而且也是一种个体与集体间的社会分 化。我愿把《跨文本跨文化》的首刊看作是在相当程度上采用这一结论性的 原则，而且就此我想提出有关权力的问题。

以各种方式提供给我的每一篇文章, 都表达了在跨文本与翻译活动中的权力问 题。作为其他文本的代表而为其存在的第二文本的权力（我使用这个名称，原 因很简单, 因为它看起来是最为中性的- - 我想强调的是此为一个基数而非序 数的名称，没有任何延迟或次等的意思），即使它如同有罪一样地隐藏起来。 这种权力不是一种赋予，亦非轻易能够获得。且不是持久和不可取消的。它恰 恰取决于其社会政治与历史背景。仍然再回到波德莱尔，问题是个体例证如何 在社会空间中被得以清晰地表达。比如说, Ratheesh Radhakrishnan有关印度影片 中电影“翻译”的探究表明, 有关电影《Aithe》德拉维族语版本的改编是如何 在一个根本不同的阶级背景中重新使之融入社会和重新创造这部影片的; 文章 透露出原版（第一版）电影的叙事方法不得不被改编，以适应一个完全不同的 社会与政治世界。可是在这场讨论中最吸引人的地方不在于对当地社会的重新 融入或独特的改编, 而是前一部影片及其改编的叙事与电影逻辑方法, 在其 断裂之中, 展示出了历史运动及其在塑造审视公众的伦理、审美与情感之技能 方面的力量。如果在Radhakrishnan的文章中有一种强烈的物质性的历史意义被 突显出来的话, 那么空间与时间的抽象, 及其被转化成地理与民族学话语理念 的翻译在Pelletier有关雷克路（Elisée Reclus）的文章中被得以强调。这篇文章认 为, 在最一般层次上一一东方与西方的断裂上, 这些话语权力的运作容易受到 
某些学者研究工作的推动, 而这些学者有关“东方” 的专长并未因其事实上 从未到过东方而变弱。因此这些认识论的和经验主义的东西存在于一种不稳定 的张力之中, 那种张力的化解只能通过愿望与权力的运作一一西方用一定的方 式 “看”东方的倾向，以及能够产生出特定事实效用的话语网络的共生权力。 费南（Villard）有关“中国”当代建构的记述令人瞩目，文章同样聚焦于现实 空间与时间的断裂，这一建构同时回到了过去，但过去又被转换成为当前最 为“现实主义的”架构。如同费南所论证的，仅仅把这看作是民族国家的共同 修正，到目前为止只能解释擦除与重新建构的特殊力量。它重又提出了有关读 者的问题，以及流入有关“中国”建构中的自负。有关过去的什么样的维度、 形态和细节看起来像是混入了那些被感知的欲望目标? 就有关过去/当前民族 国家的中国自身的建构，有关西方明确或含蓄地展示其口味、意愿和欲望的途 径而言, 这又告诉我们些什么?

表征再一次出现在Marie-Eve Blanc就法国与加拿大媒体关于越南移民之描述的比 较研究的中心。其研究表明, 描述中的差异（分别而言, 如多元文化移民的正 面形象与犯罪和玷污的负面形象）是如何依赖于巨大的社会与文化的移民背景 的。这篇文章在不同民族一国家的实践与政治框架下，解释了这些不同描述背 后的历史背景是如何发生在亚洲及其以外国家的。

蔡淑玲 (Stéphanie Shuling Tsai) 关于马尔候（Malraux）在台湾被认可情况的研究 为我们提供了一个极好的实例, 同一形象如何在其故乡之外以完全不同的方式 被接受。最为显著的是, 蔡淑玲指出了不同的敏感趋向如何影响对马尔侯有 关全球共同体观念能否被完全理解的估价。他的术语可以翻译成中文吗? 在 各种语言与文化间是否存在一种巨大的隔阂? 或者这是一个历史性的障碍, 而非语言障碍? 最后, 克汉东（Neri）的研究注意到了蔡明亮与楚浮（François Truffaut）的电影策案之间的密切关系，同样地表达了这种影响思想。克汉东指 出, 在蔡明亮影片（很显然参考了楚浮）中的互文本因素, 同样呈现了一种灵 魂的共鸣，因为两位影片制片人都被自由思想所困扰。法国新浪潮与当代台湾 电影制作间的距离因而引人注目地缩短了。作者利用这种密切关系来揭示蔡明 亮影片的特殊品质，这是一件很好的工作，与侯孝贤和杨德昌等电影制作人用 以影射欧洲和美国影片的做法形成对照。进一步向前推进使用不同的历史背景 来非常不同地影响 “自由”观念的方法是特别引人耳目的，楚浮和蔡明亮在其 影片中就利用了这样的历史背景。

就本刊的准确定位而言, 既在亚洲与欧洲的空隙之间且又向内外辐射, “现代 性的” 背景并没有散漫, 而是被问题化了。当在最初为数不多的投稿中所提出 的诸多权力问题已经继续下来的时候, 本刊文章, 以及有可能后来的投稿, 已 经证明不断增长的移民潮流、因全球化增强所引起的地方与地区间的身份断 裂、各式各样的由“外国人”转化为“国人”的接收，已经导致了更加复杂的 权力配置——如今文本的可传递性、可转码性常常依赖于相互矛盾的目标与利 益。1在这方面, 如果我要做一个恰当的建议, 那就是去了解在遗留的艺术自 治主张与社会政治历史不确定性之间的辩证张力中，翻译与跨文本表征的模式 是如何被叠加的, 而那种不确定性或许可以阻碍、推动、强迫、转变或抹擦掉 文本所处的 “跨” 形态。然而促使我们寻求从一种文本到另一种文本转化的, 不是道德愤慨或审美规范（称之为“尝试”）方面的意义，而是会保证我们评 判注意力的政治和历史的敏感性与承诺。

3 有关我早期对这些问题的思考，请参见 “Unhabituated Habituses” in David Palumbo-Liu \& Hans Ulrich Gumbrecht (eds.), Streams of Cultural Capital, Stanford, CA : Stanford University Press, 1997. 